Lowbury, E. J. L. \& HURst, L. (1956). J. gen. Microbiol. 15, 324-328

\title{
Loss of Group and Type Reactions by Tetracycline- resistant Streptococcus pyogenes
}

\author{
By E. J. L. LOWBURY AND L. HURST \\ Medical Research Council Industrial Injuries and Burns Research Unit, \\ Birmingham Accident Hospital
}

SUMMARY: Four strains of Streptococcus pyogenes were found to have lost their group and type serological reactions after acquiring resistance to tetracycline antibiotics in vitro. These degraded variants were non-haemolytic and weakly fibrinolytic; they failed to grow in fresh human blood. Some of the degraded strains were found to have acquired the property of fermenting raffinose. Antisera prepared against three degraded strains revealed some antigenic relationship between degraded forms derived from different parent strains, and between degraded and parent organisms

A variant of Streptococcus pyogenes which was resistant to the tetracyclines and would not grow aerobically on ordinary culture media has been isolated from a number of burns in this hospital and described elsewhere (Lowbury \& Hurst, 1956). We have tried without success to obtain these ' $A N$ ' forms in vitro by cultivating typical $S$. pyogenes in media containing tetracyclines. In the course of these studies, however, we found another atypical form of $S$. pyogenes which showed increased resistance to the tetracyclines and a loss of both group and type antigens. We record here some studies on these degraded variants.

\section{Strains of Streptococcus pyogenes used}

The three strains $(328,924$ and 7473$)$ which were used in most of these experiments were aerobic tetracycline-sensitive organisms isolated from burns which later yielded tetracycline-resistant anaerobic (AN) forms of $S$. pyogenes. One other strain (6357) from a burn was also used in some of the experiments.

\section{Production of degraded strains}

Methods. To obtain streptococci resistant to the tetracyclines, broth cultures of strains 328 (type 5/12/54), 924 (type 5/44) and 7473 (type 4) were subcultured by transferring one drop to each of a series of doubling dilutions of tetracycline and of oxytetracycline, separately, in nutrient broth. The highest concentration showing growth after $24 \mathrm{hr}$. at $37^{\circ}$ was subcultured to tubes containing that concentration and also twice and half that concentration of the antibiotic in broth. Subcultures were made in this way daily, the concentration of antibiotic being increased as the strains acquired resistance. Cultures were set up in duplicate, one set being incubated aerobically and the other anaerobically at $37^{\circ}$ overnight. They were subcultured on horse-blood agar, also in duplicate for aerobic and anaerobic incubation.

As an alternative method, strains 328, 924 and 6357 were inoculated in antibiotic broth as described above, and subcultured to blood agar; from blood- 
agar subcultures non-haemolytic colonies, when present, were picked to further antibiotic broth, the concentration of antibiotic being increased as the organisms acquired resistance.

Sensitivity to tetracycline and to oxytetracycline was assessed as the minimal inhibitory concentration recorded on each subculture.

Haemolysis of blood agar was recorded at each subculture, and tests for soluble haemolysin were made at the end of the series of subcultures by a standard method (see Mackie \& McCartney, 1953).

Late subcultures in the first experiment and all subcultures in the second were tested for serological group by Lancefield's method; many subcultures were also tested by Fuller's method.

Morphology was examined in Gram-stained and in nigrosin films of the original cultures and of the final subcultures.

Results. After 22 subcultures in the first experiment all the strains (3 from tetracycline and 3 from oxytetracycline) showed increased resistance to the antibiotics, the minimal inhibitory concentrations of both antibiotics rising about thirty-fold (from $0 \cdot 2-0 \cdot 4$ to $6-12 \mu \mathrm{g}$./ml.). The final subcultures failed to haemolyse blood agar or to show a soluble haemolysin; all of them had lost their group reaction but retained characteristic streptococcal morphology. Subcultures as late as the 19th, which had acquired some resistance to tetracycline, still showed the presence of group A reaction. Some of the final subcultures showed a minute colony form. All of them grew as well on aerobic as on anaerobic culture.

In the second experiment, strains 328 and 6357 , but not strain 924, yielded non-groupable, tetracycline-resistant variants which appeared within 7 days. This method (picking non-haemolytic colonies to antibiotic broth) appeared to be more reliable as well as quicker than serial subculture through antibiotic broth which, in several later experiments, failed to yield degraded streptococci.

\section{Other features of the degraded streptococci}

Fermentation reactions. Degraded forms and their parent strains were tested for fermentation of glucose, lactose, galactose, maltose, sucrose, fructose, mannitol, sorbitol, raffinose, inulin and salicin in Hiss's serum water incubated aerobically at $37^{\circ}$. Tests were examined after $18 \mathrm{hr}$. and again after $48 \mathrm{hr}$. of incubation. The parent strains fermented all the sugars except mannitol, sorbitol, raffinose and inulin. The degraded strains fermented the same sugars, but five out of six of them also fermented raffinose.

Tests for fibrinolysin. The parent organisms and degraded forms of strains 328 and 6357 were tested for fibrinolysin (streptokinase) production in the manner described by Tillett \& Garner (1933). The original strains caused complete lysis of the fibrin clot within $25 \mathrm{~min}$. and $35 \mathrm{~min}$. respectively; in this time no lysis was produced by any of the degraded strains, but partial lysis by all of them was detected after $2 \mathrm{hr}$.

Bacitracin sensitivity. Maxted (1954) showed that streptococci of group A were almost always sensitive, and streptococci of other groups almost always resistant to bacitracin. We tested the bacitracin-sensitivity of our 
original strains and of the degraded variants by a ditch plate method, with 10 units bacitracin $/ \mathrm{ml}$. in the agar ditch. There was no difference in the width of the zones of inhibition shown by the degraded forms and sensitive controls (e.g. the parent strains of Streptococcus pyogenes) inoculated on the same plate.

Mouse virulence. As the parent strains were found to be avirulent on intraperitoneal and intracerebral injection into mice, no tests were made with the degraded variants.

Growth in heparinized human blood. Tubes containing $1.0 \mathrm{ml}$. fresh heparinized human blood were inoculated each with one drop of the degraded forms of strains 328 and 6357 , grown overnight in nutrient broth, and diluted $10^{-3}$ and $10^{-5}$. A similar series of inoculations in fresh blood were made with the parent streptococci. Broth tubes were inoculated as controls. After $24 \mathrm{hr}$. at $37^{\circ}$ the tubes were examined and subcultured to blood agar. A heavy growth of the parent streptococci was found in subcultures from incubated blood, but there was no growth from either of the degraded forms after incubation in blood. All the broth controls showed a heavy growth of streptococci. The results of this experiment suggest that the degraded forms would have diminished virulence for man.

Serological typing. Parent and degraded cultures of strains 328, 924 and 7473 were typed for us by Dr W. H. H. Jebb of the Oxford Public Health Laboratory, and some also by $\operatorname{Dr}$ R. E. O. Williams at the Streptococcal Reference Laboratory, Central Public Health Laboratory, Colindale. All of the degraded strains were found to be non-typable by agglutination and precipitation methods.

Immunization of rabbits with degraded streptococci. Antisera were prepared against degraded strains 328, 914 and 7473 by intravenous inoculation of trypsinized broth cultures resuspended to $1 / 10$ of the original volume in physiological saline; $1.0 \mathrm{ml}$. of the suspension was injected on 3 consecutive days each week over a period of 4 weeks. Trypsinized vaccines were used, as recommended by McCarty \& Lancefield (1955), to facilitate the production of antisera. The rabbits were bled for serum 10 days after the last injection. Slide-agglutination tests were made with doubling dilutions (1/5 to $1 / 160)$ of these sera and of sera from unimmunized rabbits on all the degraded and all the parent streptococci. Absorption of the antisera was not attempted.

The results (see Table 1) show that the agglutinins which appeared acted not only against the immunizing strains, but also against some of the other degraded strains; two of the parent streptococci were agglutinated by one of the antisera (7473), but the other antisera did not agglutinate either the homologous or the heterologous parent strains. An unrelated strain of Streptococcus pyogenes (293) selected at random from cultures of burns was not agglutinated by any of the antisera.

Morphology. Degraded strains obtained by the first method were Grampositive cocci in long chains and were indistinguishable in morphology from the parent streptococci. By the second method the degraded organisms appeared as short chains of very small Gram-positive cocci. These differences in morphology persisted on subculture of each of the strains. 
Reversion to characteristics of the parent strain. After 50 daily subcultures in broth, two of the degraded strains (both derived from 328) were non-haemolytic and could not be grouped by Fuller's or Lancefield's methods. Similar results were obtained with shorter series of subcultures on degraded strains 924 (20 subcultures), 7473 (20 subcultures) and 6357 (30 subcultures). One culture of a degraded form of 924, which had been preserved for several weeks on Dorset egg medium was found to be giving a group A reaction and to grow in fresh heparinized human blood. For this isolated observation confirmatory material was, unfortunately, not available.

Table 1. Serology of strains of Streptococcus pyogenes degraded after growth with tetracyclines

\section{DISCUSSION}

The ease with which streptococci of group A were found to lose their group and type reactions as well as their haemolytic properties in the presence of tetracyclines suggests a mechanism by which these organisms may escape detection and possibly persist in the host tissues after apparently successful chemotherapy. The altered forms, however, had probably lost virulence, as they failed to grow in fresh human blood and produced little fibrinolysin; moreover, they did not revert to the typical form after 20-50 subcultures in the absence of tetracyclines. The good results on skin grafting of burns after elimination of Streptococcus pyogenes by a tetracycline (Lowbury \& Cason, 1954) suggest that degraded streptococci, if they are present in burns after a course of the antibiotic, do not exert any local pathogenic effect. The possibility of a reversion to virulent forms cannot, however, be excluded and is suggested by an isolated observation reported above.

Gezon (1948) reported acquired resistance in streptococci of group A grown in the presence of penicillin. Such strains also showed, in some instances, a loss of group antigen, and a loss of mouse virulence was always found. Sensitivity was restored by serial subculture on penicillin-free medium, and virulence was 
occasionally restored by passage in normal mice. Similar results were reported with strains grown in the presence of chlortetracycline (Gezon \& Fasan, 1950). The phenomenon described by these workers is probably the same as that observed by us, and it is interesting that it can be induced by different and unrelated antibiotics.

The antigenic change associated with acquired resistance to tetracyclines in our experiments contrasts with the persistence of group and type antigens and of virulence in the tetracycline-resistant ' $\mathrm{AN}$ ' forms isolated from burns (Lowbury \& Hurst, 1956). It differs, too, from the changes described by Wilson (1945) on serial passage of type 27 streptococci in mice; in these experiments the $\mathrm{C}$ polysaccharide of the streptococcus was lost, but type specificity persisted.

Differences in the morphology and in the serological behaviour of degraded organisms have suggested that these constitute a heterogeneous group, but some antigenic relationship between different degraded strains and between degraded and parent strains was also apparent.

We are much indebted to Dr R. E. O. Williams, Director of the Streptococcal Reference Laboratory, Colindale, and to Drs R. W. Vollum and W. H. H. Jebb of the Public Health Laboratory, Oxford, for valuable suggestions and for serological examination of our strains.

\section{REFERENCES}

Gezon, H. M. (1948). Antibiotic studies on $\beta$ haemolytic streptococci. I. Proc. Soc. exp. Biol. N.Y. 67, 208.

Gezon, H. M. \& Fasan, D. M. (1950). Antibiotic studies on $\beta$ haemolytic streptococci. VI. Proc. Soc. exp. Biol., N.Y. 73, 10.

Lowbury, E. J. L. \& Cason, J. S. (1954). Aureomycin and erythromycin therapy for Strep. pyogenes in burns. Brit. Med. J. ii, 914.

Lowbury, E. J. L. \& HURST, L. (1956). Atypical anaerobic forms of Strep. pyogenes resistant to the tetracyclines. J. clin. Path. 9, 59.

McCarty, M. \& LANCEFiELD, R. C. (1955). Variation in the group specific carbohydrate of group A streptococci. J. exp. Med. 102, 11.

Mackie, T. J. \& McCantney, J. E. (1953). Handbook of Practical Bacteriology, 9th ed., p. 336. Edinburgh: E. and S. Livingstone.

Maxted, W. R. (1954). The use of bacitracin for identifying group A haemolytic streptococci. J. clin. Path. 6, 224.

Tilnetr, W. S. \& Garner, R. L. (1933). The fibrinolytic activity of haemolytic streptococci. J. exp. Med. 58, 485.

Wilson, A. T. (1945). Loss of group carbohydrate during mouse passages of a group A haemolytic streptococcus. J. exp. Med. 81, 593.

(Received 26 March 1956) 